\title{
Set Theoretic Methods in Model Predictive Control
}

\author{
Saša V. Raković
}

\begin{abstract}
The main objective of this paper is to highlight the role of the set theoretic analysis in the model predictive control synthesis. In particular, the set theoretic analysis is invoked to: (i) indicate the fragility of the model predictive control synthesis with respect to variations of the terminal constraint set and the terminal cost function and (ii) discuss a simple, tube based, robust model predictive control synthesis method for a class of nonlinear systems.
\end{abstract}

Keywords: Control Synthesis, Set Invariance, Tube Model Predictive Control.

\section{Introduction}

The contemporary research has recognized the need for an adequate mathematical framework permitting the meaningful robust control synthesis for constrained control systems. An appropriate framework to address the corresponding robust control synthesis problems is based on the utilization of the set theoretic analysis, see, for instance, a partial list of pioneering contributions [1-4] and comprehensive monographs [5, 6] for a detailed overview. A set of alternative but complementary control synthesis methods utilizing game-theoretic approaches is also studied [7, 8].

The robust model predictive control synthesis problem is one of the most important and classical problems in model predictive control [9, 10]. The power of the set theoretic analysis has been already utilized in the tube model predictive control synthesis [11-15] and the characterization of the minimal invariant sets [16, 17]. The main objective of this paper is to indicate a further role of the set theoretic analysis in the model predictive control synthesis $[9,12,18]$.

Saša V. Raković

Honorary Research Associate at the Centre for Process Systems Engineering, Imperial College London, SW7 2AZ, UK

and

Scientific Associate at the Institute for Automation Engineering of Otto-von-Guericke-Universität Magdeburg, Germany

e-mail: svresasavrakovic.com 


\section{Outline of the paper}

Section 2 introduces systems under considerations and highlights the role of information available for the control synthesis. Section 3 collects some basic notions, definitions and results relevant for the control synthesis under constraints and uncertainty. Section 4 recalls basic results of the receding horizon control synthesis and utilizes the set theoretic analysis to indicate fragility of the receding horizon control. Section 5 proposes a simple, tube based, robust model predictive control synthesis for a particular class of non-linear systems. Section 6 provides concluding remarks.

\section{Nomenclature and Basic Definitions}

The sets of non-negative, positive integers and non-negative real numbers are denoted, respectively, by $\mathbb{N}, \mathbb{N}_{+}$and $R_{+}$, i.e. $\mathbb{N}:=\{0,1,2, \ldots\}, \mathbb{N}_{+}:=\{1,2, \ldots\}$ and $R_{+}:=\{x \in R: x \geq 0\}$. For $q_{1}, q_{2} \in \mathbb{N}$ such that $q_{1}<q_{2}$ we denote $\mathbb{N}_{\left[q_{1}: q_{2}\right]}:=$ $\left\{q_{1}, q_{1}+1, \ldots, q_{2}-1, q_{2}\right\}$. For two sets $X \subset R^{n}$ and $Y \subset R^{n}$, the Minkowski set addition is defined by $X \oplus Y:=\{x+y: x \in X, y \in Y\}$ and the Minkowski set subtraction is $X \ominus Y:=\left\{z \in R^{n}: z \oplus Y \subseteq X\right\}$. For a set $X \subset R^{n}$ and a vector $x \in R^{n}$ we write $x \oplus X$ instead of $\{x\} \oplus X$. A set $X \subset R^{n}$ is a $C$ set if it is compact, convex, and contains the origin. A set $X \subset R^{n}$ is a proper $C$ set if it is a $C$ set and the origin is in its non-empty interior. A set $X \subseteq R^{n}$ is a symmetric set (with respect to the origin in $R^{n}$ ) if $X=-X$. We denote by $|x|_{L}$ norm of the vector $x$ induced by a symmetric, proper $C$ set $L$. For sets $X \subset R^{n}$ and $Y \subset R^{n}$, the Hausdorff semi-distance and the Hausdorff distance of $X$ and $Y$ are, respectively, given by:

$$
\begin{aligned}
& h_{L}(X, Y):=\inf _{\alpha}\{\alpha: X \subseteq Y \oplus \alpha L, \alpha \geq 0\} \text { and } \\
& H_{L}(X, Y):=\max \left\{h_{L}(X, Y), h_{L}(Y, X)\right\}
\end{aligned}
$$

where $L$ is a given, symmetric, proper $C$ set in $R^{n}$. Given a function $f(\cdot), f^{k}(x), k \in$ $\mathbb{N}$ stands for its $k$-th iterate at the point $x$, i.e $f^{k}(x)=f\left(f^{k-1}(x)\right)=f\left(f\left(f^{k-2}(x)\right)\right)=$ .... If $f(\cdot)$ is a set-valued function from, say, $X$ into $U$, namely, its values are subsets of $U$, then its graph is the set $\{(x, y): x \in X, y \in f(x)\}$.

\section{System Description and Role of Information}

In the deterministic case, the variables inducing the dynamics are the state $z \in R^{n}$ and the control $v \in R^{m}$. The underlying dynamics in the deterministic case is discretetime and time-invariant and is generated by a mapping $\bar{f}(\cdot, \cdot): R^{n} \times R^{m} \rightarrow R^{n}$. When the current state and control are, respectively, $z$ and $v$, then:

$$
z^{+}=\bar{f}(z, v)
$$

is the successor state. The system variables, i.e. the state $z$ and the control $v$ are subject to hard constraints:

$$
z \in \mathbb{Z} \text { and } v \in \mathbb{V}
$$


where sets $\mathbb{Z}$ and $\mathbb{V}$ are, respectively, subsets of $R^{n}$ and $R^{m}$. Likewise, in the basic uncertain model, the variables inducing the dynamics are the state $x \in R^{n}$, the control $u \in R^{m}$ and the disturbance $w \in R^{p}$. The considered dynamics is discrete-time and time-invariant and is generated by a mapping $f(\cdot, \cdot, \cdot): R^{n} \times R^{m} \times R^{p} \rightarrow R^{n}$. As in the basic deterministic model, when the current state, control and disturbance are, respectively, $x, u$ and $w$, then:

$$
x^{+}=f(x, u, w)
$$

is the successor state. The system variables, i.e. the state $x$, the control $u$ and the disturbance $w$ are subject to hard constraints:

$$
x \in \mathbb{X}, u \in \mathbb{U} \text { and } w \in \mathbb{W},
$$

where $\mathbb{X}, \mathbb{U}$ and $\mathbb{W}$ are, respectively, subsets of $R^{n}, R^{m}$ and $R^{p}$. In this paper we invoke the following technical assumption:

Assumption 1. (i) The function $\bar{f}(\cdot, \cdot): R^{n} \times R^{m} \rightarrow R^{n}$ is continuous and sets $\mathbb{Z}$ and $\mathbb{V}$ are compact. (ii) The function $f(\cdot, \cdot, \cdot): R^{n} \times R^{m} \times R^{p} \rightarrow R^{n}$ is continuous and sets $\mathbb{X}, \mathbb{U}$ and $\mathbb{W}$ are compact.

An additional ingredient playing a crucial role in the uncertain case is the one of the information available for the control synthesis.

Interpretation 1 (Inf-Sup Type Information). At any time $k$ when the decision concerning the control input $u_{k}$ is taken, the state $x_{k}$ is known while the disturbance $w_{k}$ is not known and can take arbitrary value $w_{k} \in \mathbb{W}$.

Under Interpretation 11 at any time instance $k$, the feedback rules $u_{k}=u_{k}\left(x_{k}\right)$ are allowed.

Interpretation 2 (Sup-Inf Type Information). At any time $k$ when the decision concerning the control input $u_{k}$ is taken, both the state $x_{k}$ and the disturbance $w_{k} \in$ $\mathbb{W}$ are known while future disturbances $w_{k+i}, i \in \mathbb{N}_{+}$are not known and can take arbitrary values $w_{k+i} \in \mathbb{W}, i \in \mathbb{N}_{+}$.

Clearly, under Interpretation 2 the feedback rules $u_{k}=u_{k}\left(x_{k}, w_{k}\right)$ are also, in addition to the feedback rules $u_{k}=u_{k}\left(x_{k}\right)$, allowed at any time instance $k$.

\section{Constrained Controllability}

An important role of the set theoretic analysis in the control synthesis is the characterization of controllability sets under constraints and uncertainty. A very simple, natural and basic problem, in the control synthesis in the deterministic case, is: Given a target set $\mathbb{T} \subseteq \mathbb{Z}$, characterize the set of all states $z \in \mathbb{Z}$, say $\mathbb{S}$, and all control laws $v(\cdot): \mathbb{S} \rightarrow \mathbb{V}$ such that for all $z \in \mathbb{S}$ and a control law $v(\cdot)$ it holds that $\bar{f}(z, v(z)) \in \mathbb{T}$. Obviously, similar questions can be posed, in a transparent way, for both variants, i.e. inf-sup and sup-inf variants, of control synthesis in the uncertain case. We indicate the mathematical formalism providing answers to these 
basic questions and obtained by a direct utilization of the set theoretic analysis. We consider in the deterministic case, for a given, non-empty, set $Z \subseteq \mathbb{Z}$, the preimage mapping $\overline{\mathscr{B}}(\cdot)$ and the set-valued control map $\mathscr{V}(\cdot)$ given by:

$$
\begin{aligned}
& \overline{\mathscr{B}}(Z):=\{z: \exists v \in \mathbb{V} \text { such that } \bar{f}(z, v) \in Z\} \cap \mathbb{Z} \text { and } \\
& \forall z \in \overline{\mathscr{B}}(Z), \mathscr{V}(z):=\{v \in \mathbb{V}: \bar{f}(z, v) \in Z\} .
\end{aligned}
$$

Similarly, for a given, non-empty, set $X \subseteq \mathbb{X}$, under Interpretation 1 , the inf-sup preimage mapping $\mathscr{B}_{\text {inf-sup }}(\cdot)$ and the inf-sup set-valued control map $\mathscr{U}_{\text {inf-sup }}(\cdot)$ are given by:

$$
\begin{aligned}
& \mathscr{B}_{\text {inf-sup }}(X):=\{x: \exists u \in \mathbb{U} \text { such that } \forall w \in \mathbb{W}, f(x, u, w) \in X\} \cap \mathbb{X} \text { and } \\
& \forall x \in \mathscr{B}_{\text {inf-sup }}(X), \mathscr{U}_{\text {inf-sup }}(x):=\{u \in \mathbb{U}: \forall w \in \mathbb{W}, f(x, u, w) \in X\} .
\end{aligned}
$$

Likewise, under Interpretation 2, the sup-inf preimage mapping $\mathscr{B}_{\text {sup-inf }}(\cdot)$ and the sup-inf set-valued control map $\mathscr{U}_{\text {sup-inf }}(\cdot, \cdot)$ are given by:

$$
\begin{aligned}
& \mathscr{B}_{\text {sup-inf }}(X):=\{x: \forall w \in \mathbb{W}, \exists u \in \mathbb{U} \text { such that } f(x, u, w) \in X\} \cap \mathbb{X} \text { and } \\
& \forall(x, w) \in \mathscr{B}_{\text {sup-inf }}(X) \times \mathbb{W}, \mathscr{U}_{\text {sup-inf }}(x, w):=\{u \in \mathbb{U}: f(x, u, w) \in X\} .
\end{aligned}
$$

Evidently, given a non-empty set $Z \subseteq \mathbb{Z}$, the set of states that are one step controllable to $Z$ is the set $\overline{\mathscr{B}}(Z)$ and any control law $v(\cdot): \overline{\mathscr{B}}(Z) \rightarrow \mathbb{V}$ ensuring that the successor state $\bar{f}(z, v(z))$ is in the set $Z$ is a selection of the set-valued control map $\mathscr{V}(\cdot)$. Similarly, the set of states that are one step inf-sup controllable to $X$ is the set $\mathscr{B}_{\text {inf-sup }}(X)$ and any control law $u(\cdot): \overline{\mathscr{B}}(X) \rightarrow \mathbb{U}$ ensuring that all possible successor states $f(x, u(x), w), w \in \mathbb{W}$ are in the set $X$ is a selection of the inf-sup set-valued control map $\mathscr{U}_{\text {inf-sup }}(\cdot)$. Likewise, the set of states that are one step sup-inf controllable to $X$ is the set $\mathbb{S}_{\text {sup-inf }}=\mathscr{B}_{\text {sup-inf }}(X)$ and any control law $u(\cdot, \cdot): \overline{\mathscr{B}}(X) \times \mathbb{W} \rightarrow \mathbb{U}$ ensuring that any successor state $f(x, u(x, w), w)$ is in the set $X$ is a selection of the sup-inf set-valued control map $\mathscr{U}_{\text {sup-inf }}(\cdot, \cdot)$. If Assumption 1 (i) holds and a target set $Z$ is compact, the set $\overline{\mathscr{B}}(Z)$ and the graph of the set-valued control map $\mathscr{V}(\cdot)$ are compact when non-empty. Likewise, if Assumption 1 (ii) holds and a target set $X$ is compact then the set $\mathscr{B}_{\text {inf-sup }}(X)$ and the graph of the inf-sup set-valued control map $\mathscr{U}_{\text {inf }- \text { sup }}(\cdot)$ are compact when nonempty and, similarly, the set $\mathscr{B}_{\text {sup-inf }}(X)$ and the graph of the sup-inf set-valued control map $\mathscr{U}_{\text {sup-inf }}(\cdot, \cdot)$ are compact when non-empty. The semi-group property of preimage mappings permits the characterization of the $N$-step, the $N$-step infsup and the $N$-step sup-inf controllable sets and corresponding set-valued control maps by dynamic programming procedures indicated next. Let $N$ be an arbitrary integer and let $\mathbb{T}$ be a given target set. The $N$-step controllable sets and corresponding set-valued control maps are given in the deterministic case, for $j \in \mathbb{N}_{[1: N]}$, by:

$$
\begin{aligned}
\mathscr{Z}_{j} & :=\overline{\mathscr{B}}\left(\mathscr{Z}_{j-1}\right) \text { and } \forall z \in \mathscr{Z}_{j}, \\
\mathscr{V}_{j}(z) & :=\left\{v \in \mathbb{V}: \bar{f}(z, v) \in \mathscr{Z}_{j-1}\right\},
\end{aligned}
$$


with the boundary condition $\mathscr{Z}_{0}:=\mathbb{T} \subseteq \mathbb{Z}$. Similarly, the $N$-step inf-sup controllable sets and corresponding inf-sup set-valued control maps are given, for $j \in \mathbb{N}_{[1: N]}$, by:

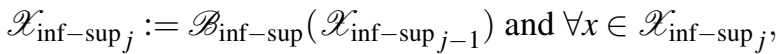

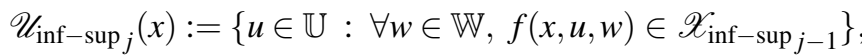

with the boundary condition $\mathscr{X}_{\text {inf-sup }}:=\mathbb{T} \subseteq \mathbb{X}$. Likewise, the $N$-step sup-inf controllable sets and corresponding sup-inf set-valued control maps are given, for $j \in \mathbb{N}_{[1: N]}$, by:

$$
\begin{aligned}
& \mathscr{X}_{{\text {sup }- \text { inf }_{j}}_{j}}:=\mathscr{B}_{\text {sup-inf }}\left(\mathscr{X}_{\text {sup }- \text { inf }_{j-1}}\right) \text { and } \forall(x, w) \in \mathscr{X}_{{\text {sup }- \text { inf }_{j}}} \times \mathbb{W} \text {, }
\end{aligned}
$$

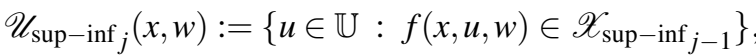

with the boundary condition $\mathscr{X}_{\text {sup }-\inf _{0}}:=\mathbb{T} \subseteq \mathbb{X}$. If Assumption 1 holds and a target set $\mathbb{T}$ is compact then: (i) the $k$-step controllable set $\mathscr{Z}_{k}$ satisfies $\mathscr{Z}_{k}=\overline{\mathscr{B}}^{k}(\mathbb{T})$ and $\mathscr{Z}_{k}$ and the graph of the set-valued control map $\mathscr{V}_{k}(\cdot)$ are compact when non-empty; (ii) the $k$-step inf-sup controllable set $\mathscr{X}_{\text {inf-sup }_{k}}$ satisfies $\mathscr{X}_{\text {inf-sup }}=\mathscr{B}_{\text {inf-sup }}^{k}(\mathbb{T})$ and $\mathscr{X}_{\text {inf-sup }}$ and the graph of the inf-sup set-valued control map $\mathscr{U}_{\text {inf-sup }}(\cdot)$ are compact when non-empty; and (iii) the $k$-step sup-inf controllable set $\mathscr{X}_{\text {sup-inf }}$ satisfies $\mathscr{X}_{\text {sup-inf }}=\mathscr{B}_{\text {sup-inf }}^{k}(\mathbb{T})$ and $\mathscr{X}_{\text {sup-inf }}$ and the graph of the sup-inf set-valued control map $\mathscr{U}_{\text {sup-inf }}(\cdot, \cdot)$ are compact when non-empty. It is well known that the non-emptiness of the $N$-step, the $N$-step inf-sup and the $N$-step sup-inf controllable sets represents, respectively, necessary and sufficient conditions for solvability of the $N$-step, the $N$-step inf-sup and the $N$-step sup-inf controllability to a target set control problems [2, 4-6]. Likewise, the afore mentioned non-emptiness plays a crucial role in the solvability of the finite horizon (of length $N$ ) optimal and robust (inf-sup and sup-inf) optimal control problems in the presence of terminal set constraints (as is the case in the receding horizon control [9]).

A further role of preimage mappings is also evident in set invariance [5, 6]:

Definition 1. A set $Z$ is a control invariant set for the system $z^{+}=\bar{f}(z, v)$ and constraints set $(\mathbb{Z}, \mathbb{V})$ if and only if $Z \subseteq \overline{\mathscr{B}}(Z)$.

$A$ set $X$ is an inf-sup control invariant set for the system $x^{+}=f(x, u, w)$ and constraints set $(\mathbb{X}, \mathbb{U}, \mathbb{W})$ if and only if $X \subseteq \mathscr{B}_{\mathrm{inf}-\sup }(X)$.

A set $X$ is a sup-inf control invariant set for the system $x^{+}=f(x, u, w)$ and constraints set $(\mathbb{X}, \mathbb{U}, \mathbb{W})$ if and only if $X \subseteq \mathscr{B}_{\text {sup-inf }}(X)$.

A more subtle issue is related to properties of fixed points of preimage mappings. The fixed point set equations of preimage mappings take the form:

$$
X=\overline{\mathscr{B}}(X), X=\mathscr{B}_{\text {inf-sup }}(X) \text { and } X=\mathscr{B}_{\text {sup }- \text { inf }}(X),
$$

where the unknown, in any of the three cases, is the set $X$. It is known [5, 6] that, under Assumption 11 special fixed points of preimage mappings are the maximal 
control invariant set $\bar{\Omega}_{\infty}$, the maximal inf-sup control invariant set $\Omega_{\infty}^{\text {inf-sup }}$ and the maximal sup-inf control invariant set $\Omega_{\infty}^{\text {sup-inf }}$ given, respectively, by:

$$
\bar{\Omega}_{\infty}=\bigcap_{k=0}^{\infty} \overline{\mathscr{B}}^{k}(\mathbb{Z}), \Omega_{\infty}^{\mathrm{inf}-\text { sup }}=\bigcap_{k=0}^{\infty} \mathscr{B}_{\mathrm{inf}-\text { sup }}^{k}(\mathbb{X}), \text { and } \Omega_{\infty}^{\text {sup }- \text { inf }}=\bigcap_{k=0}^{\infty} \mathscr{B}_{\text {sup }- \text { inf }}^{k}(\mathbb{X}) .
$$

Under Assumption 1 sets $\bar{\Omega}_{\infty}, \Omega_{\infty}^{\text {inf-sup }}$ and $\Omega_{\infty}^{\text {sup-inf }}$ are compact, when non-empty, and are, in fact, unique maximal (with respect to set inclusion) fixed points of corresponding preimage mappings $\overline{\mathscr{B}}(\cdot), \mathscr{B}_{\text {inf }- \text { sup }}(\cdot)$ and $\mathscr{B}_{\text {sup-inf }}(\cdot)$.

\section{Fragility of Receding Horizon Control}

We indicate fragility of the receding horizon control by utilizing the set theoretic analysis and exploiting the fact that fixed points of preimage mappings are, in general, non-unique. Given an integer $N \in \mathbb{N}$ let $\mathbf{v}_{N}:=\left\{v_{0}, v_{1}, \ldots, v_{N-1}\right\}$ denote the control sequence of length $N$, let also $\phi\left(i, z, \mathbf{v}_{N}\right)$ denote the solution of (1) at time $i \in \mathbb{N}_{[0: N]}$ when the initial state at time 0 is $z$ and the control sequence is $\mathbf{v}_{N}$. The cost function $V_{N}(\cdot, \cdot): \mathbb{Z} \times \mathbb{V}^{N} \rightarrow R_{+}$is specified by:

$$
V_{N}\left(z, \mathbf{v}_{N}\right):=\sum_{i=0}^{N-1} \ell\left(\phi\left(i ; z, \mathbf{v}_{N}\right), v_{i}\right)+V_{f}\left(\phi\left(N ; z, \mathbf{v}_{N}\right),\right.
$$

where functions $\ell(\cdot, \cdot): \mathbb{Z} \times \mathbb{V} \rightarrow R_{+}$and $V_{f}(\cdot): \mathbb{Z}_{f} \rightarrow R_{+}$are the path and terminal cost and $\mathbb{Z}_{f} \subseteq \mathbb{Z}$ is the terminal constraint set. Let also

$$
\begin{gathered}
\mathbb{V}_{N}(z):=\left\{\mathbf{v}_{N} \in \mathbb{V}^{N}: \forall i \in \mathbb{N}_{[0, N-1]},\left(\phi\left(i ; z, \mathbf{v}_{N}\right), v_{i}\right) \in \mathbb{Z} \times \mathbb{V}\right. \text { and } \\
\left.\phi\left(N ; z, \mathbf{v}_{N}\right) \in \mathbb{Z}_{f}\right\},
\end{gathered}
$$

denote the set of admissible control sequences at an initial condition $z \in \mathbb{Z}$. We invoke usual assumptions employed in the model predictive control [9]:

Assumption 2. (i) The function $\bar{f}(\cdot, \cdot)$ satisfies $0=\bar{f}(0,0)$. (ii) The terminal constraint set $\mathbb{Z}_{f} \subseteq \mathbb{Z}$ is a compact set and $(0,0) \in \mathbb{Z}_{f} \times \mathbb{V}$. (iii) The path and terminal cost functions $\ell(\cdot, \cdot): \mathbb{Z} \times \mathbb{V} \rightarrow R_{+}$and $V_{f}(\cdot): \mathbb{Z}_{f} \rightarrow R_{+}$are continuous, $\ell(0,0)=0$ and $V_{f}(0)=0$ and there exist positive scalars $c_{1}, c_{2}, c_{3}$ and $c_{4}$ such that for all $(z, v) \in \mathbb{Z} \times \mathbb{V}$ it holds that $c_{1}|z|^{2} \leq \ell(z, v) \leq c_{2}$ and for all $x \in \mathbb{Z}_{f}$ it holds that $c_{3}|z|^{2} \leq V_{f}(z) \leq c_{4}|z|^{2}$ (iv) There exists a control law $\kappa_{f}(\cdot): \mathbb{Z}_{f} \rightarrow \mathbb{V}$ such that for all $z \in \mathbb{Z}_{f}$ it holds that $\bar{f}\left(z, \kappa_{f}(z)\right) \in \mathbb{Z}_{f}$ and $V_{f}\left(\bar{f}\left(z, \kappa_{f}(z)\right)\right)+\ell\left(z, \kappa_{f}(z)\right) \leq V_{f}(z)$.

Given an integer $N \in \mathbb{N}$, we consider the optimal control problem $\mathbb{P}_{N}(z)$ :

$$
\begin{aligned}
\mathbb{P}_{N}(z): \quad V_{N}^{*}(z):=\min _{\mathbf{v}_{N}}\left\{V_{N}\left(z, \mathbf{v}_{N}\right): \mathbf{v}_{N} \in \mathbb{V}_{N}(z)\right\} \\
\mathbf{v}_{N}^{*}(z):=\arg \min _{\mathbf{v}_{N}}\left\{V_{N}\left(z, \mathbf{v}_{N}\right): \mathbf{v}_{N} \in \mathbb{V}_{N}(z)\right\} .
\end{aligned}
$$


In the model predictive control, the optimal control problem $\mathbb{P}_{N}(z)$ is solved online at the state $z$, encountered in the process, and the optimizing control sequence $\mathbf{v}_{N}^{*}(z)=\left\{v_{0}^{*}(z), v_{1}^{*}(z), \ldots, v_{N-1}^{*}(z)\right\}$ is utilized to obtain the model predictive control law by applying its first term $v_{0}^{*}(z)$ (or its selection when $\mathbf{v}_{N}^{*}(z)$ is set-valued) to the system (1). The domain of the value function $V_{N}^{*}(\cdot)$ and the optimizing control sequence $\mathbf{v}_{N}^{*}(\cdot)$ is given by:

$$
\mathscr{Z}_{\mathrm{MPC} N}:=\left\{z: \mathbb{V}_{N}(z) \neq \emptyset\right\} .
$$

At the conceptual level, in the deterministic case, the model predictive control law is an on-line implementation of the receding horizon control law; The explicit form of the receding horizon control law and the value function can be obtained by solving the optimal control problem $\mathbb{P}_{N}(z)$ utilizing parametric mathematical programming techniques [19] or by employing parametric mathematical programming in conjunction with the standard dynamic programming procedure given, for each $j \in \mathbb{N}_{[1: N]}$, by:

$$
\begin{aligned}
\mathscr{Z}_{j} & :=\overline{\mathscr{B}}\left(\mathscr{Z}_{j-1}\right), \quad \forall z \in \mathscr{Z}_{j}, \mathscr{V}_{j}(z):=\left\{v \in \mathbb{V}: \bar{f}(z, v) \in \mathscr{Z}_{j-1}\right\}, \\
\forall z \in \mathscr{Z}_{j}, V_{j}^{*}(z) & :=\min _{v}\left\{\ell(z, v)+V_{j-1}^{*}(\bar{f}(z, v)): v \in \mathscr{V}_{j}(z)\right\} \\
\forall z \in \mathscr{Z}_{j}, \kappa_{j}^{*}(z) & :=\arg \min _{v}\left\{\ell(z, v)+V_{j-1}^{*}(\bar{f}(z, v)): v \in \mathscr{V}_{j}(z)\right\}, \text { with } \\
\mathscr{Z}_{0} & :=\mathbb{Z}_{f} \text { and } \forall z \in \mathscr{Z}_{0}, V_{0}^{*}(z):=V_{f}(z) .
\end{aligned}
$$

Obviously, qualitative system theoretic properties of the model predictive control and the receding horizon control are equivalent. Under Assumptions 1 and 2, the origin is an exponentially stable attractor for the controlled system, possibly setvalued system when $\kappa_{N}^{*}(\cdot)$ is set-valued,:

$$
\forall z \in \mathscr{Z}_{N}, z^{+} \in \bar{F}(z):=\left\{\bar{f}(z, \tilde{v}): \tilde{v} \in \kappa_{N}^{*}(z)\right\},
$$

with the basin of attraction being equal to a compact set $\mathscr{Z}_{N}=\overline{\mathscr{B}}^{N}\left(\mathbb{Z}_{f}\right)$. The corresponding stability property is, in fact, a strong property, that is it holds for all state trajectories $\left\{z_{k}\right\}_{k=0}^{\infty}$ satisfying (17). The first role of set theoretic analysis is the characterization of the domain $\mathscr{Z}_{N}$ of the value function $V_{N}^{*}(\cdot)$ and the receding horizon control law $\kappa_{N}^{*}(\cdot)$; The corresponding domain is the set $\overline{\mathscr{B}}^{N}\left(\mathbb{Z}_{f}\right)$ which is, under Assumptions 1 and 2 compact for any fixed integer $N \in \mathbb{N}$ and, additionally, an invariant set (i.e. $\forall z \in \mathscr{Z}_{N}, \bar{F}(z) \subseteq \mathscr{Z}_{N}$ ). Clearly, it is, then, of interest to understand how is the domain of functions $V_{N}^{*}(\cdot)$ and $\kappa_{N}^{*}(\cdot)$ affected by the variation of the terminal constraint set $\mathbb{Z}_{f}$ (and possibly variation of the terminal cost function $\left.V_{f}(\cdot)\right)$. A crucial point in understanding this important issue is closely related to properties of the preimage mapping $\overline{\mathscr{B}}(\cdot)$ and, in fact, non-uniqueness and attractivity properties of its fixed points. We now deliver a simple example illustrating the fragility of the receding horizon control. 
Example 1. Consider the two dimensional system generated by a linear map:

$$
\bar{f}(z, v)=\frac{1}{2} I z+I v, \text { so that } z^{+}=\frac{1}{2} I z+I v,
$$

with constraints on system variables $z \in R^{2}$ and $v \in R^{2}$ :

$$
\mathbb{Z}=[-4,4] \times\left[-2^{l}, 2^{l}\right] \text { and } \mathbb{V}=[-1,1] \times\{0\} \text { with } l \in \mathbb{N} .
$$

The maximal control invariant set is, clearly, the set $\mathbb{Z}$ as:

$$
\mathbb{Z}=\overline{\mathscr{B}}(\mathbb{Z}) \text { and, consequently, } \mathbb{Z}=\overline{\mathscr{B}}^{N}(\mathbb{Z}) \text { for any integer } N \in \mathbb{N} \text {. }
$$

However, the set $Z:=[-4,4] \times\{0\}$ is also a fixed point of the mapping $\overline{\mathscr{B}}(\cdot)$ :

$$
Z=\overline{\mathscr{B}}(Z) \text { and, consequently, } Z=\overline{\mathscr{B}}^{N}(Z) \text { for any integer } N \in \mathbb{N} \text {. }
$$

A moment of reflection reveals that for any compact set $Y$ such that $0 \in Y \subseteq Z$ :

$$
[-2,2] \times\{0\} \subseteq \overline{\mathscr{B}}(Y) \subseteq[-4,4] \times\{0\} \text { and } \overline{\mathscr{B}}(Y) \subseteq \overline{\mathscr{B}}^{k+1}(Y)=[-4,4] \times\{0\},
$$

for any integer $k \in \mathbb{N}_{+}$. A further examination shows that for any compact set $X$ such that $\{0\} \times[-2 \varepsilon, 2 \varepsilon]=X \subseteq \mathbb{Z}$ (with $\varepsilon>0$ arbitrarily small) we have:

$$
\begin{aligned}
& X \subseteq[-2,2] \times[-4 \varepsilon, 4 \varepsilon] \cap \mathbb{Z}=\overline{\mathscr{B}}(X) \subseteq[-4,4] \times[-4 \varepsilon, 4 \varepsilon] \cap \mathbb{Z}, \\
& \forall k \in \mathbb{N}_{+}, \overline{\mathscr{B}}^{k}(X) \subseteq \overline{\mathscr{B}}^{k+1}(X)=[-4,4] \times\left[-2^{k+2} \varepsilon, 2^{k+2} \varepsilon\right] \cap \mathbb{Z}, \text { and } \\
& \text { for all } k \in \mathbb{N} \text { such that } k \geq 2 \text { and } 2^{k+1} \varepsilon \geq 2^{l}, \overline{\mathscr{B}}^{k}(X)=\mathbb{Z} .
\end{aligned}
$$

A variant of the example relevant to the receding horizon control follows. Pick an integer $N \in \mathbb{N}$ and consider the receding horizon control synthesis problem with the following ingredients. The path cost function is:

$$
\ell(z, v)=z^{\prime} Q z+v^{\prime} R v \text { with } Q=\frac{14}{16} I \text { and } R=I,
$$

The terminal cost function is the unconstrained infinite horizon value function:

$$
V_{f}(z)=z^{\prime} I z .
$$

The corresponding unconstrained infinite horizon optimal control law is:

$$
\kappa_{f}(z)=-\frac{1}{4} I z
$$

and the terminal constraint set $\mathbb{Z}_{f}$ is the maximal positively invariant set for the system $z^{+}=\frac{1}{4} I z$ subject to constraints $z \in \mathbb{Z}$ and $\frac{1}{4} I z \in \mathbb{V}$ which turns out to be the set $Z=[-4,4] \times\{0\}$. All assumptions (our Assumptions 1 1 and 2) commonly employed in the model predictive control literature [9] are satisfied. Unfortunately, 
the set $\mathbb{Z}_{f}=Z=[-4,4] \times\{0\}$ is a fixed point of the preimage mapping $\overline{\mathscr{B}}(\cdot)$ so that $\mathbb{Z}_{f}=\overline{\mathscr{B}}^{N}\left(\mathbb{Z}_{f}\right)$ for any integer $N$. In turn, regardless of the choice of the horizon length $N \in \mathbb{N}$, the receding horizon control law $\kappa_{N}^{*}(\cdot)$ and the corresponding value function $V_{N}^{*}(\cdot)$ are defined only over the set $\mathscr{Z}_{N}=\overline{\mathscr{B}}^{N}\left(\mathbb{Z}_{f}\right)=\mathbb{Z}_{f}=Z$, which is a compact, zero measure, subset of the maximal control invariant set $\mathbb{Z}$. The exact constrained infinite horizon control value function and control law are given by:

$$
\forall z \in \mathbb{Z}, V_{\infty}^{*}(z)=z^{\prime}\left(\begin{array}{cc}
1 & 0 \\
0 & \frac{14}{12}
\end{array}\right) z, \text { and } \kappa_{\infty}^{*}(z)=\left(\begin{array}{cc}
-\frac{1}{4} & 0 \\
0 & 0
\end{array}\right) z .
$$

In fact, in our example, the following, fixed-point type, relations hold true:

$$
\begin{aligned}
& \mathbb{Z}=\overline{\mathscr{B}}(\mathbb{Z}), \forall z \in \mathbb{Z}, \mathscr{V}_{\infty}(z)=\{v \in \mathbb{V}: \bar{f}(z, v) \in \mathbb{Z}\} \neq \emptyset, \\
& \forall z \in \mathbb{Z}, V_{\infty}^{*}(z)=\min _{v}\left\{\ell(z, v)+V_{\infty}^{*}(\bar{f}(z, v)): v \in \mathscr{V}_{\infty}(z)\right\}, \\
& \forall z \in \mathbb{Z}, \kappa_{\infty}^{*}(z)=\arg \min _{v}\left\{\ell(z, v)+V_{\infty}^{*}(\bar{f}(z, v)): v \in \mathscr{V}_{\infty}(z)\right\} .
\end{aligned}
$$

Choosing the terminal constraint set $\mathbb{Z}_{f}=[-4,4] \times[-2 \varepsilon, 2 \varepsilon] \subseteq \mathbb{Z}$, with $\varepsilon>0$, and the terminal cost function $V_{f}(\cdot)=V_{\infty}^{*}(\cdot)$ leads (for a sufficiently large horizon length $N$, for example for $N \in \mathbb{N}$ such that $2^{N+1} \varepsilon \geq 2^{l}$ ) to the receding horizon control law $\kappa_{N}^{*}(\cdot)$ and the value function $V_{N}^{*}(\cdot)$ identically equal over the whole set $\mathbb{Z}$ to the infinite horizon control law $\kappa_{\infty}^{*}(\cdot)$ and the infinite horizon value function $V_{\infty}^{*}(\cdot)$. Hence an $\varepsilon>0$ variation of the ingredients (the set $\mathbb{Z}_{f}$ and the function $V_{f}(\cdot)$ ) for the receding horizon control synthesis results in a discontinuous change of domains of the corresponding receding horizon control law $\kappa_{N}^{*}(\cdot)$ and the corresponding value function $V_{N}^{*}(\cdot)$ (notice that the Hausdorff distance between sets $Z=[-4,4] \times\{0\}$ and $\mathbb{Z}=[-4,4] \times\left[-2^{l}, 2^{l}\right]$ can be made as large as we please by setting $l$ large enough).

Conclusion 1. Standard assumptions employed in the model predictive control [9] (summarized by Assumptions 1 and 2) are not, in the general case, sufficient assumptions to ensure convergence (as $N \rightarrow \infty$ ) of the receding horizon control law $\kappa_{N}^{*}(\cdot)$, the value function $V_{N}^{*}(\cdot)$ and the corresponding domain $\mathscr{Z}_{N}$ to the infinite horizon control law $\kappa_{\infty}^{*}(\cdot)$, the value function $V_{\infty}^{*}(\cdot)$ and the corresponding domain denoted $Z_{\infty}$. Furthermore, an $\varepsilon>0$ variation of the terminal constraint set $\mathbb{Z}_{f}$ and the terminal cost function $V_{f}(\cdot)$, such that the perturbed data satisfy usual assumptions can result, in general case, in the discontinuous change of the domain $\mathscr{Z}_{N}$ of the receding horizon control law $\kappa_{N}^{*}(\cdot)$ and the corresponding value function $V_{N}^{*}(\cdot)$; Hence the receding horizon control synthesis is fragile, even in the linear-polytopic case, with respect to feasible perturbations of the terminal constraint set $\mathbb{Z}_{f}$ and the terminal cost function $V_{f}(\cdot)$.

\section{Simple Tube Model Predictive Control}

The potential structure of the underlying mapping $f(\cdot, \cdot, \cdot)$ generating the dynamics is beneficial for the simplified inf-sup tube model predictive control synthesis, as 
illustrated in [11-14] for special classes of discrete time systems (including linear, piecewise affine and some classes of nonlinear systems). Ideas employed in [1114] are, now, demonstrated by considering a class of non-linear systems (that has interesting structure and has not been treated in [11-15]) for which:

$$
f(x, u, w)=g(x)+B u+w \text { so that } x^{+}=g(x)+B u+w .
$$

With the uncertain system (18) we associate a nominal system generated by:

$$
\bar{f}(z, v)=g(z)+B v \text { so that } z^{+}=g(z)+B v,
$$

and work in this subsection under the following simplifying assumption:

Assumption 3. There exists a function $\theta(\cdot, \cdot): R^{n} \times R^{n} \rightarrow R^{m}$ such that:

(i) for all $x$ and $z,|g(x)-g(z)+B \theta(x, z)|_{L} \leq \lambda|x-z|_{L}$ for some $\lambda \in[0,1)$; (ii) for all $x$ and $z$ such that $|x-z|_{L} \leq \gamma$, where $\gamma:=(1-\lambda)^{-1} \mu$ and $\mu:=h_{L}(\mathbb{W},\{0\})$, it holds that $|\theta(x, z)|_{M} \leq \eta$; (iii) for all $x \in \gamma L$ and $y \in \gamma L, \mid g(x)+B \theta(x, 0)-g(y)-$ $\left.B \theta(y, 0)\right|_{L} \leq \lambda^{*}|x-y|_{L}$ for some $\lambda^{*} \in[0, \lambda] \subset[0,1)$.

Since $|g(x)+B(v+\theta(x, z))+w-g(z)-B v|_{L} \leq \lambda|x-z|_{L}+|w|_{L}$ by Assumption3(i), and, since $\lambda(1-\lambda)^{-1} h_{L}(\mathbb{W},\{0\})+h_{L}(\mathbb{W},\{0\})=(1-\lambda)^{-1} h_{L}(\mathbb{W},\{0\})$, the following simple but useful fact is affirmative:

Lemma 1. Suppose Assumptions $[$ ( $i$ ) and $[$ (ii) hold and consider a set $X:=z \oplus \gamma L$ where $z \in R^{n}, \gamma:=(1-\lambda)^{-1} \mu$ and $\mu:=h_{L}(\mathbb{W},\{0\})$. Then for all $x \in X$ and all $v \in R^{m}$ it holds that $\theta(x, z) \in \eta M$ and $g(x)+B(v+\theta(x, z)) \oplus \mathbb{W} \subseteq z^{+} \oplus \gamma L$ with $z^{+}=g(z)+B v$.

Lemma 1 motivates the use of the parameterized inf-sup tube-control policy pair. The parameterized tube $\mathbf{X}_{\text {inf-sup }}$ is the sequence of sets $\left\{X_{\text {inf-sup }_{k}}\right\}_{k=0}^{N}$ where:

$$
\forall k \in \mathbb{N}_{[0: N]}, X_{\text {inf- }-\sup _{k}}:=z_{k} \oplus \gamma L \text {. }
$$

The corresponding parameterized policy $\Pi_{\mathrm{inf}-\sup _{N}}$ is the sequence of control laws $\left\{\pi_{\text {inf-sup }}(\cdot, \cdot)\right\}_{k=0}^{N-1}$ where:

$$
\forall k \in \mathbb{N}_{[0: N-1]}, \forall y \in X_{\text {inf }-\sup _{k}}, \pi_{\text {inf }-\sup _{k}}\left(y, z_{k}\right):=v_{k}+\theta\left(y, z_{k}\right) .
$$

To exploit fully Lemma 1 we need an additional and mild assumption:

Assumption 4. Sets $\mathbb{Z}:=\mathbb{X} \ominus \gamma L$ and $\mathbb{V}:=\mathbb{U} \ominus \eta M$ are non-empty and such that $(0,0) \in \mathbb{Z} \times \mathbb{V}$.

A direct argument exploiting mathematical induction and Lemma 1 yields:

Proposition 1. Suppose Assumptions 3 (i), 3 (ii) and Assumption 4 hold. Assume also that sequences $\left\{z_{k}\right\}_{k=0}^{N}$ and $\left\{v_{k}\right\}_{k=0}^{N-1}$ are such that $z_{0} \in \mathbb{Z}$ and, for all $k \in$ $\mathbb{N}_{[0: N-1]}, z_{k+1}=g\left(z_{k}\right)+B v_{k} \in \mathbb{Z}$ and $v_{k} \in \mathbb{V}$. Consider the parameterized tube-

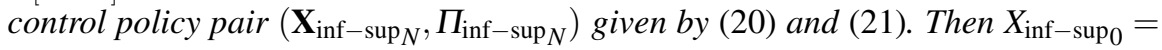
$z_{0} \oplus \gamma L \subseteq \mathbb{Z} \oplus \gamma L \subseteq \mathbb{X}$ and for all $k \in \mathbb{N}_{[0: N-1]}$ it holds that: 


$$
\begin{aligned}
& \forall y \in X_{\text {inf }-\sup _{k}}, \pi_{\text {inf }-\sup _{k}}\left(y, z_{k}\right)=v_{k}+\theta\left(y, z_{k}\right) \in \mathbb{V} \oplus \eta M \subseteq \mathbb{U}, \\
& X_{\text {inf }-\sup _{k+1}}=z_{k+1} \oplus \gamma L \subseteq \mathbb{Z} \oplus \gamma L \subseteq \mathbb{X} \text {, and },
\end{aligned}
$$

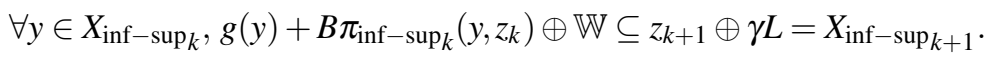

We now provide a more general result under an additional assumption and utilize it in conjunction with Proposition 1 for the tube model predictive control.

Assumption 5. There exists a compact set $\mathscr{Z}_{N} \subseteq \mathbb{Z}$ with $0 \in \mathscr{Z}_{N}$ and functions $\kappa_{N}^{*}(\cdot): \mathscr{Z}_{N} \rightarrow \mathbb{V}$ with $\kappa_{N}^{*}(0)=0$ and $V_{N}^{*}(\cdot): \mathscr{Z}_{N} \rightarrow R_{+}$with $V_{N}^{*}(0)=0$ such that: (i) For all $z \in \mathscr{Z}_{N}$ it holds that $z^{+}=g(z)+B \kappa_{N}^{*}(z) \in \mathscr{Z}_{N}$; (ii) The origin is exponentially stable for the controlled system $z^{+}=g(z)+B \kappa_{N}^{*}(z)$ with the basin of attraction $\mathscr{Z}_{N}$, i.e. all sequences $\left\{z_{k}\right\}_{k=0}^{\infty}$ with arbitrary $z_{0} \in \mathscr{Z}_{N}$ and $z_{k+1}=g\left(z_{k}\right)+B \kappa_{N}^{*}\left(z_{k}\right)$ satisfy $\left|z_{k}\right|_{L} \leq \alpha^{k} \beta\left|z_{0}\right|_{L}$ for some $\alpha \in[0,1)$ and $\beta \in[0, \infty)$; (iii) The function $V_{N}^{*}(\cdot)$ is lower semi-continuous over the set $\mathscr{Z}_{N}$, continuous at the origin and it induces the property assumed above in (ii).

For all $x \in \mathscr{Z}_{N} \oplus \gamma L$ let $\mathscr{Z}(x):=\left\{z \in \mathscr{Z}_{N}:(x-z) \in \gamma L\right\}$ and define:

$$
\begin{aligned}
& \forall x \in \mathscr{Z}_{N} \oplus \gamma L, V_{N}^{0}(x):=\min _{z}\left\{V_{N}^{*}(z): z \in \mathscr{Z}(x)\right\}, \text { and } \\
& \forall x \in \mathscr{Z}_{N} \oplus \gamma L, z^{0}(x):=\arg \min _{z}\left\{V_{N}^{*}(z): z \in \mathscr{Z}(x)\right\} .
\end{aligned}
$$

We consider the feedback control law and the corresponding induced controlled uncertain system given by:

$$
\begin{array}{r}
\forall x \in \mathscr{Z}_{N} \oplus \gamma L, \kappa_{N}^{0}(x):=\kappa_{N}^{*}\left(z^{0}(x)\right)+\theta\left(x, z^{0}(x)\right) \text { and } \\
\forall x \in \mathscr{Z}_{N} \oplus \gamma L, x^{+} \in F(x):=\left\{g(x)+B \kappa_{N}^{0}(x)+w: w \in \mathbb{W}\right\},
\end{array}
$$

A straight-forward utilization of Lemma 1 and construction above yields:

Theorem 1. Suppose Assumptions 1 , 3 (i), 3 (ii), 4 and 5 hold. Then: (i) for all $x \in \mathscr{Z}_{N} \oplus \gamma L$ it holds that $\mathscr{Z}(x) \neq \emptyset$ and for any $x \in \mathscr{Z}_{N} \oplus \gamma L$ there exists at least one $z \in \mathscr{Z}(x)$ such that $V_{N}^{*}(z)=V_{N}^{0}(x)$; (ii) for all $x \in z \oplus \gamma L$ with arbitrary $z \in \mathscr{Z}_{N}$ it holds that $V_{N}^{0}(x)=V_{N}^{*}\left(z^{0}(x)\right) \leq V_{N}^{*}(z)$; (iii) for all $x \in \gamma L$ it holds that $V_{N}^{0}(x)=0$, $z^{0}(x)=0, \kappa_{N}^{0}(x)=\theta(x, 0)$ and $g(x)+B \kappa_{N}^{0}(x) \oplus \mathbb{W} \subseteq \gamma L ;$ (iv) For all state sequences $\left\{x_{k}\right\}_{k=0}^{\infty}$ with arbitrary $x_{0} \in \mathscr{Z}_{N} \oplus \gamma L$ and generated by (23) it holds that, for all $k$, :

$$
\begin{aligned}
& x_{k} \in z^{0}\left(x_{k}\right) \oplus \gamma L \subseteq \mathscr{Z}_{N} \oplus \gamma L \subseteq \mathbb{X}, \\
& \kappa_{N}^{0}\left(x_{k}\right)=\kappa_{N}^{*}\left(z^{0}\left(x_{k}\right)\right)+\theta\left(x_{k}, z^{0}\left(x_{k}\right)\right) \in \mathbb{V} \oplus \eta M \subseteq \mathbb{U}, \\
& V_{N}^{0}\left(x_{k+1}\right)=V_{N}^{*}\left(z^{0}\left(x_{k+1}\right)\right) \leq V_{N}^{*}\left(g\left(z^{0}\left(x_{k}\right)\right)+B \kappa_{N}^{*}\left(z^{0}\left(x_{k}\right)\right)\right), \\
& h_{L}\left(z^{0}\left(x_{k}\right) \oplus \gamma L, \gamma L\right) \leq \alpha^{k} \beta\left|z^{0}\left(x_{0}\right)\right|_{L} \text { and } h_{L}\left(\left\{x_{k}\right\}, \gamma L\right) \leq \alpha^{k} \beta\left|z^{0}\left(x_{0}\right)\right|_{L},
\end{aligned}
$$

for some scalars $\alpha \in[0,1)$ and $\beta \in[0, \infty)$.

Under Assumptions 1 and 3 a direct application of results in [16, Section 4] yields that the mapping $\tilde{F}(X):=\{g(x)+B \theta(x, 0)+w: x \in X, w \in \mathbb{W}\}$ is a contraction 
on the space of compact subsets of $\gamma L$ (note that Assumption 3 implies $\tilde{F}(\gamma L) \subseteq \gamma L$ ) and it admits the unique fixed point, namely there exists a compact subset $\mathbb{O}$ of $\gamma L$ such that $\mathbb{O}=\tilde{F}(\mathbb{O})$ and iterates $\tilde{F}^{k+1}(\gamma L) \subseteq \tilde{F}^{k}(\gamma L)$ converge, with respect to the Hausdorff distance, exponentially fast to the set $\mathbb{O}$ as $k \rightarrow \infty$. Hence, in addition to assertions of Theorem 1, we have:

Corollary 1. Suppose Assumptions 1, 3, 4 and 5hold. Then there exists a compact subset $\mathbb{O}$ of $\gamma L$ such that $\left\{g(x)+B \kappa_{N}^{0}(x)+w: x \in \mathbb{O}, w \in \mathbb{W}\right\}=\mathbb{O}$ where $\kappa_{N}^{0}(\cdot)$ is given by (23). Furthermore, for all state sequences $\left\{x_{k}\right\}_{k=0}^{\infty}$ with arbitrary $x_{0} \in$ $\mathscr{Z}_{N} \oplus \gamma L$ and generated by (23) it holds that, for all $k, h_{L}\left(\left\{x_{k}\right\}, \mathbb{O}\right) \leq \tilde{\alpha}^{k} \tilde{\beta}\left|z^{0}\left(x_{0}\right)\right|_{L}$ for some scalars $\tilde{\alpha} \in[0,1)$ and $\tilde{\beta} \in[0, \infty)$.

It should be clear that the set $\mathscr{Z}_{N}$ and functions $\kappa_{N}^{*}(\cdot): \mathscr{Z}_{N} \rightarrow \mathbb{V}$ and $V_{N}^{*}(\cdot)$ : $\mathscr{Z}_{N} \rightarrow R_{+}$appearing in Assumption 5 and utilized in (22) and (23), Theorem 1 and Corollary 1 can be obtained implicitly, under Assumptions 1 , 4 and 2 by the standard model predictive control synthesis considered in Section 4 (namely, functions $\kappa_{N}^{*}(\cdot)$ and $V_{N}^{*}(\cdot)$ can be computed implicitly by solving $\mathbb{P}_{N}(z)$, specified in (14), online and the set $\mathscr{Z}_{N}$ is given, implicitly, by 15 or alternatively by $\mathscr{Z}_{N}=\overline{\mathscr{B}}^{N}\left(\mathbb{Z}_{f}\right)$ where $\mathbb{Z}_{f} \subseteq \mathbb{Z}$ is the corresponding terminal constraint set utilized in (14)). Utilizing Proposition 1 and the implicit representation of the set $\mathscr{Z}_{N}$, given in (15), and functions $\kappa_{N}^{*}(\cdot)$ and $V_{N}^{*}(\cdot)$, obtained from (14), we provide a formulation of an optimal control problem that when solved on-line provides the implementation of the parameterized tube receding horizon control law (23). Given an integer $N \in \mathbb{N}$, the corresponding parameterized tube optimal control problem $\mathbb{P}_{\text {tube } N}(x)$ is:

$$
\begin{aligned}
\mathbb{P}_{\text {tube } N}(x): \quad V_{N}^{0}(x) & :=\min _{\left(z, \mathbf{v}_{N}\right)}\left\{V_{N}\left(z, \mathbf{v}_{N}\right): \mathbf{v}_{N} \in \mathbb{V}_{N}(z),(x-z) \in \gamma L\right\} \\
\left(z, \mathbf{v}_{N}\right)^{0}(x) & :=\arg \min _{\left(z, \mathbf{v}_{N}\right)}\left\{V_{N}\left(z, \mathbf{v}_{N}\right): \mathbf{v}_{N} \in \mathbb{V}_{N}(z),(x-z) \in \gamma L\right\} .
\end{aligned}
$$

Note that the tube model predictive control problem $\mathbb{P}_{\text {tube } N}(x)$ is marginally more complex than the conventional model predictive control problem $\mathbb{P}_{N}(z)$, specified in (14), as it includes $z$ as an additional decision variable and has an additional constraint $(x-z) \in \gamma L$ which, by construction, can be satisfied for all $x \in \mathscr{Z}_{N} \oplus \gamma L$. The control applied to the system $x^{+}=g(x)+B u+w, w \in \mathbb{W}$ at the state $x \in \mathscr{Z}_{N} \oplus$ $\gamma L$, encountered in the process, is given by:

$$
\kappa_{N}^{0}(x)=v_{0}^{0}(x)+\theta\left(x, z^{0}(x)\right)
$$

It is, in fact, the on-line implicit implementation of the feedback utilized in 23) and, hence, it ensures, under Assumptions 1, 3, 4 and 2, that properties established in Theorem 1 and Corollary 1 hold for the controlled uncertain system given by $\forall x \in \mathscr{Z}_{N} \oplus \gamma L, x^{+} \in\left\{g(x)+B\left(v_{0}^{0}(x)+\theta\left(x, z^{0}(x)\right)\right)+w: w \in \mathbb{W}\right\}$.

Remark 1. All results established above are applicable to the sup-inf case with direct modifications. One of many possible and simple synthesis methods for the sup-inf case would require only changes in Assumption 3 (i.e. the use of function $\theta(\cdot, \cdot, \cdot)$ rather than $\theta(\cdot, \cdot)$ and direct modifications of remaining parts of 
Assumption 3) and the utilization of the parameterized sup-inf tube-control policy pair $\left(\mathbf{X}_{\text {sup-inf }}, \Pi_{\text {sup-inf }}\right)$ where, as in (20) and (21), for any $k$, we employ $X_{\text {sup-inf }}:=z_{k} \oplus \gamma L$ and for any $(y, \tilde{w}) \in X_{\text {sup }-\inf _{k}} \times \mathbb{W}$, we consider parameterized control laws $\pi_{\text {sup-inf }}\left(y, \tilde{w}, z_{k}\right):=v_{k}+\theta\left(y, \tilde{w}, z_{k}\right)$.

\section{Concluding Remarks}

We highlighted the role of the set theoretic analysis in the model predictive control synthesis and suggested that it provides qualitative insights that are beneficial for the receding horizon control synthesis. We indicated the fragility of the model predictive control and proposed a simple tube model predictive control synthesis method for a particular class of non-linear systems.

Acknowledgements. The author is grateful to E. Crück, S. Olaru and H. Benlaoukli for research interactions leading to an ongoing, collaborative, research project on the fragility of the receding horizon control.

\section{References}

1. Witsenhausen, H.S.: A minimax control problem for sampled linear systems. IEEE Transactions on Automatic Control 13, 5-21 (1968)

2. Bertsekas, D.P., Rhodes, I.B.: On the Minimax Reachability of Target Sets and Target Tubes. Automatica 7, 233-247 (1971)

3. Glover, J.D., Schweppe, F.C.: Control of Linear Dynamic Systems with Set Constrained Disturbances. IEEE Transactions on Automatic Control 16(5), 411-423 (1971)

4. Kurzhanski, A.B.: Control and Estimation Under Uncertainty. Nauka, Moscow (1977) (in Russian)

5. Aubin, J.P.: Viability Theory. Birkhäuser, Basel (1991)

6. Blanchini, F., Miani, S.: Set-Theoretic Methods in Control. Birkhäuser, Basel (2008)

7. Krasovski, N.N., Subbotin, A.I.: Game-theoretical Control Problems. Springer, New York (1988)

8. Başar, T., Olsder, J.G.: Dynamic noncooperative game theory. Academic Press, New York (1995)

9. Mayne, D.Q., Rawlings, J.B., Rao, C.V., Scokaert, P.O.M.: Constrained Model Predictive Control: Stability and Optimality. Automatica 36(6), 789-814 (2000)

10. Magni, L., De Nicolao, G., Scattolini, R., Allgöwer, F.: Robust Model predictive Control of Nonlinear Discrete-Time Systems. International Journal of Robust and Nonlinear Control 13(3-4), 229-246 (2003)

11. Raković, S.V., Mayne, D.Q.: Robust model predictive control of constrained piecewise affine discrete time systems. In: Proceedings of the 6th IFAC Symposium - NOLCOS 2004, Stuttgart, Germany (September 2004)

12. Raković, S.V., Mayne, D.Q.: Set Robust Control Invariance for Linear Discrete Time Systems. In: Proceedings of the 44th IEEE CDC and ECC conference CDCECC 2005, Sevilla, Spain (2005)

13. Mayne, D.Q., Seron, M.M., Raković, S.V.: Robust model predictive control of constrained linear systems with bounded disturbances. Automatica 41(2), 219-224 (2005) 
14. Raković, S.V., Teel, A.R., Mayne, D.Q., Astolfi, A.: Simple Robust Control Invariant Tubes for Some Classes of Nonlinear Discrete Time Systems. In: Proceedings of the 45th IEEE CDC Conference, CDC 2006, San Diego, USA (December 2006)

15. Mayne, D.Q., Kerrigan, E.C.: Tube-Based Robust Nonlinear Model Predictive Control. In: Proceedings of the 7th IFAC Symposium - NOLCOS 2007, Pretoria, South Africa (August 2007)

16. Artstein, Z., Raković, S.V.: Feedback and Invariance Under Uncertainty via Set-Iterates. Automatica 44(2), 520-525 (2008)

17. Raković, S.V.: Minkowski Algebra and Banach Contraction Principle in Set Invariance for Linear Discrete Time Systems. In: Proceedings of 46th IEEE CDC Conference, CDC 2007, New Orleans, USA (2007)

18. Barić, M., Raković, S.V., Besselmann, T., Morari, M.: Max-Min Optimal Control of Constrained Discrete-Time Systems. In: Proceedings of 17th IFAC World Congress, IFAC 2008, Seoul, Korea (2008)

19. Bank, B., Guddat, J., Klatte, D., Kummer, B., Tammer, K.: Non-linear Parametric Optimization. Birkhäuser, Basel (1983) 